\title{
Optical rotatory power, biaxiality, and models of chiral tilted smectic phases
}

\author{
V. P. Panov, ${ }^{1}$ J. K. Vij, ${ }^{1, *}$ N. M. Shtykov, ${ }^{1, \dagger}$ S. S. Seomun, ${ }^{1}$ D. D. Parghi, ${ }^{2}$ M. Hird, ${ }^{2}$ and J. W. Goodby ${ }^{2}$ \\ ${ }^{1}$ Institute of Advanced Materials Science and Department of Electronic Engineering, Trinity College, University of Dublin, \\ Dublin 2, Ireland \\ ${ }^{2}$ Department of Chemistry, University of Hull, Hull HU6 7RX, United Kingdom
}

(Received 14 February 2003; published 11 August 2003)

\begin{abstract}
Among the chiral tilted smectics, the stable existence has been confirmed in numerous investigations of $\mathrm{Sm}_{A}{ }^{*}$, (antiferroelectric smectic- $\left.C_{A}\right) \mathrm{Sm}_{\mathrm{F} 11}{ }^{*}\left(\mathrm{Sm}_{\gamma^{*}}{ }^{*}\right), \mathrm{Sm} C_{\mathrm{F} 12} *$ (antiferroelectric, $\mathrm{AF}$ ) and $\mathrm{Sm} C^{*}$ phases. The structures of the ferrielectric $\mathrm{SmC}_{\mathrm{F} 11}$ * and $\mathrm{Sm} C_{\mathrm{F} 12} *$ phases suggested by different models are essentially different although all the models use the three-layer and four-layer periodicity for them. The structures of the phases were investigated using the optical rotatory power (ORP) measurements technique. The ORP was simulated using Berreman's $4 \times 4$-matrix method. The compound under investigation (S)-1-methylheptyl 4-(4'-n-undecyloxy-biphenyl-4-yl-carbonyloxy) [acronym (S)-11OF1M7] clearly provides $\mathrm{SmC}_{\mathrm{F} 11}$ * and $\mathrm{SmC} C_{\mathrm{F} 12}{ }^{*}$ phases, the temperature range for the existence of these phases is about $5{ }^{\circ} \mathrm{C}$ each. This had not been achieved for the earlier investigated antiferroelectric liquid crystal (AFLC) samples. The results obtained confirm that the unit cell of the molecular structure of these subphases is highly biaxial. Due to the biaxiality the texture of the homeotropic cell under a polarizing microscope appears nonuniform. This requires a special approach to the measurements and a simulation of the ORP, which is discussed in detail. A technique has been designed where the transmitted intensity through a polarizing microscope is measured as a function of the angle of polarization of the incident light. From the observed output, which is a biased sine wave, the ORP is being determined. In the same scan, the wavelength of light is also being automatically altered. Comparing the simulated and measured data, we can conclude that in the $\mathrm{SmC}_{\mathrm{F} 12}{ }^{*}$ phase the distortion angle of the directors in the Ising model is lower than $10^{\circ}$. Using the Ising model, the pitch in $\mathrm{SmC}_{\mathrm{F} 11}{ }^{*}$ has been determined and this is found to have a strong temperature dependence.
\end{abstract}

DOI: 10.1103/PhysRevE.68.021702

\section{INTRODUCTION}

Several different theoretical approaches [1-4] have been advanced for explaining a variety of the chiral phases between paraelectric smectic- $A\left(\mathrm{Sm} A^{*}\right)$ and antiferroelectric smectic- $C_{A}\left(\mathrm{SmC}_{A}{ }^{*}\right)$. The phase sequence is generally presented as follows: $\mathrm{SmC}_{A} *-\mathrm{FI}_{L}(\operatorname{spr} 3)-\mathrm{SmC}_{\mathrm{F} 11}$ * $\left(\mathrm{SmC}_{\gamma}{ }^{*}, \mathrm{SmC}_{3}\right)-\mathrm{FI}_{H} \quad(\operatorname{spr} 2)-\mathrm{SmC}_{\mathrm{F} 12} *\left(\mathrm{AF}, \mathrm{SmC} C_{4}\right)-$ $\mathrm{SmC}_{\mathrm{F} 13}{ }^{*}$ (FiLC, $\left.\operatorname{spr} 1\right)-\mathrm{Sm} C^{*}-\mathrm{Sm} C_{\alpha}{ }^{*}-\mathrm{Sm} A^{*}$ [5].

Until recently, the uniaxial clock model [3] was strongly suggested by the resonant $\mathrm{x}$-ray scattering observations $[6,7]$. However, it contradicted the results of the optical measurements [8]. Meanwhile, one-dimensional Ising model for explaining the layer structure of the phase is based on ferroelectric and antiferroelectric ordering of the neighboring smectic layers in terms of the Ising spins [9]. This model can explain the optical biaxiality observed in the various phases [8].

The results of ellipsometric experiment on freely suspended ferrielectric films [10] strongly support the deformed (or distorted) model and this has also recently been supported by the higher resolution resonant $\mathrm{x}$-ray experiments [11]. This approach is used in the present work.

The observation of the Schlieren textures in the freestanding films [12] confirms the large biaxiality of a unit cell in

\footnotetext{
*Author to whom correspondence should be addressed. Email address: jvij@tcd.ie

${ }^{\dagger}$ Permanent address: Institute of Crystallography, Russian Academy of Sciences, Leninsky prosp. 59, 117333 Moscow, Russia.
}

PACS number(s): 61.30.-v, 64.70.Md, 42.70.Df

the phases. The recent studies of the optical rotatory power (ORP) [13-15] provide an efficient method for a quantitative analysis of the unit-cell structure.

Nevertheless, it is shown here that the large biaxiality found in the phases leads to an inevitable optical nonuniformity of the texture of a homeotropically aligned sample. This should, therefore, influence the results of the ORP measurements.

The effect of the optical nonuniformity in the texture is discussed in detail. The measurements using a wide beam of light allow us to overcome this effect during the experiment. Averaging of the transmitted intensity over the initial azimuthal angle $\varphi_{0}$ of the molecules is needed for carrying out the computer simulations.

In conjunction with a new material, which provides $\mathrm{SmC}_{\mathrm{F} 12} *$ and $\mathrm{SmC}_{\mathrm{F} 11}$ * phases over wider temperature ranges than hitherto studied, the results allow us to make a significant step towards the understanding of the structure of the phases discussed.

\section{EXPERIMENTAL PROCEDURE}

The material (S)-1-methylheptyl 4-(4'-n-undecyloxybiphenyl-4-yl-carbonyloxy) [acronym $(S)$-11OF1M7] [sometimes known as $(S)$-11OBP1M7] was synthesized in Hull, UK. Despite a slight disagreement in the phase transition temperatures [16] determined by different methods, it clearly provides $\mathrm{Sm} C_{\mathrm{F} 12}$ * and $\mathrm{Sm} C_{\mathrm{F} 11}$ * phases, which exist over a temperature range of about $5{ }^{\circ} \mathrm{C}$ each. The temperature range found for $\mathrm{SmC}_{\mathrm{F} 11}$ * in this material is probably the widest known to date. 
The chemical formula of the compound is given as

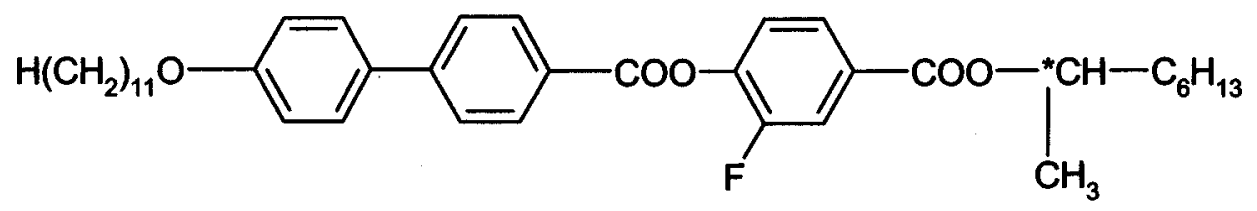

Different experiments give different phase transitions temperatures. This is explained by the differences in the cooling rates, the surface conditions, the prehistory of the sample, and the hysteresis in the phase transition temperatures. Since in the temperature range that extends from $\mathrm{SmC} C^{*}$ to $\mathrm{SmC}_{A} *$ phases, the system is frustrated [17], some relatively weak additional effects may significantly affect the emergence of phases and temperatures of phase transitions. This requires that in a single temperature scan the optical rotatory power measurements must be carried out. As a reference, the following phase sequence can be used:

Crystal $\left(28^{\circ} \mathrm{C}\right) \quad \mathrm{SmC}_{A} *\left(64^{\circ} \mathrm{C}\right) \mathrm{SmC}_{\mathrm{F} 11^{*}}\left(68.3^{\circ} \mathrm{C}\right)$ $\mathrm{Sm} C_{\mathrm{F} 12}{ }^{*}\left(76.9^{\circ} \mathrm{C}\right) \mathrm{Sm}_{\mathrm{F} 13}{ }^{*}\left(85^{\circ} \mathrm{C}\right) \mathrm{Sm} C^{*}\left(88^{\circ} \mathrm{C}\right) \mathrm{Sm} A^{*}$ $\left(107.4^{\circ} \mathrm{C}\right)$ Isotropic.

To overcome the hysteresis in the phase transition temperatures, the setup for optical rotatory power measurements [13] has been modified to allow for the entire set of measurements to be carried out during a single temperature scan, as required. The experimental arrangement is given in Fig. 1. The light passes a computer-controlled block of filters, polarizer, the sample, and then the analyzer. The transmitted intensity is measured by a photodiode, the output is fed to an oscilloscope which is triggered by the reference signal from the generator. During the experiment, the polarizer is designed to rotate at a speed of $\sim 600 \mathrm{sec}^{-1}$ and the output of the detector is fed to the oscilloscope and the oscillograms are recorded by the computer.

The output signal as a function of the angle of polarization can be expressed in terms of a biased sine wave. The

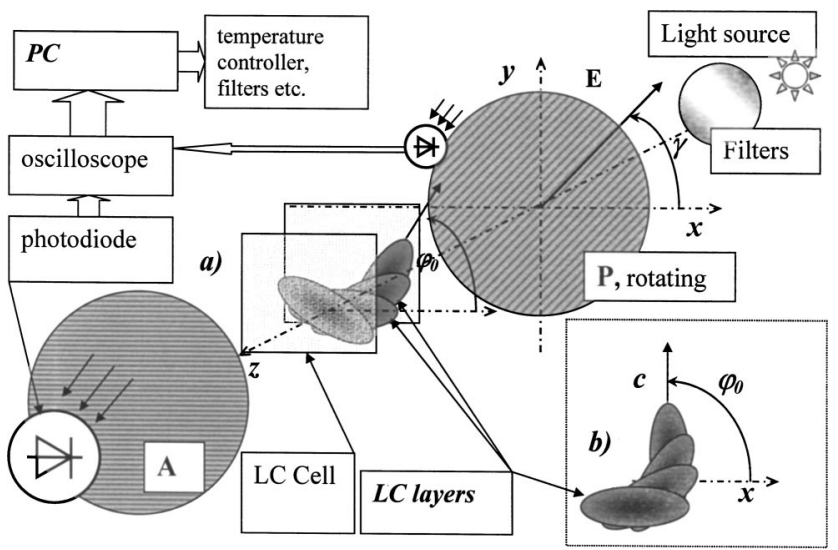

FIG. 1. The setup for the ORP measurements (the temperature control system is not shown) and the definitions of the parameters. Here, (a) and (b) are the two possible cases of the molecular (or c director) arrangement. oscillograms captured during the measurements were recorded and fitted to the biased sine wave function in order to obtain the phase, the amplitude, and the bias. It will be shown in the section on theory that the phase of the output sine wave will correspond to the double optical rotation angle. The other two parameters of a sine wave, the amplitude and the bias, contain information about the ellipticity and the intensity of the transmitted light, as shown later.

The measurements were performed using sandwich cells of thicknesses 8-60 $\mu \mathrm{m}$ in which homeotropic alignment was achieved using orientating films of carboxylatochromium complex (chromolane). These were spin coated onto the glass substrates. The surfaces were cured for time duration of $30 \mathrm{~min}$ at a temperature of $120^{\circ} \mathrm{C}$ and then used without rubbing. The cell was heated, filled with the compound in its isotropic phase and slowly cooled to the $\operatorname{Sm} A$ * phase. The textures of the different smectic phases were observed with a polarizing microscope.

\section{THEORY}

Since the output light, in general, is elliptically polarized, the ORP can be determined using the theory of the method given below. When the light propagates through a linear layered media, the expression for the transmitted wave can be presented as follows:

$$
\left(\begin{array}{l}
T_{x} \\
T_{y}
\end{array}\right)=\left(\begin{array}{cc}
t_{x x} & t_{y x} \\
t_{x y} & t_{y y}
\end{array}\right)\left(\begin{array}{c}
E_{x} \\
E_{y}
\end{array}\right),
$$

where $E_{x}, E_{y}, T_{x}, T_{y}$, are the $x$ and $y$ components of the electric field of the incident, transmitted, and reflected waves, respectively. The coefficients $t_{x x}, t_{y x}, t_{x y}, t_{y y}$ are the elements of the matrix that connects the electric field vectors of $\mathbf{E}$ and $\mathbf{T}$.

The simplest way to obtain these coefficients is to use Jones $2 \times 2$-matrix method. However, this method neglects the reflected wave and, as shown in Ref. [14], cannot be used for simulating the multilayered systems that possesses selective reflection.

The Jones matrices are essential unitary and Hermitian. This means that

$$
\begin{gathered}
t_{x x}=t_{y y}^{*}, \\
t_{y x}=-t_{x y}^{*}, \\
\left|t_{x x}\right|^{2}+\left|t_{x y}\right|^{2}=1 .
\end{gathered}
$$


The asterisk in Eq. (2) represents the complex conjugate of the number. For the case when conditions given by a set of Eq. (2) are satisfied, the calculation of optical rotation from the elements of this matrix is straightforward.

However, the exact solution of the problem requires the solution using a $4 \times 4$-matrix method. Here, we use the classical method given by Berreman [18]. In this case, the matrix elements in Eq. (1) do not necessarily meet the conditions given by a set of equations (2) since the $4 \times 4$-matrix method includes the reflection and the interference of the reflected waves. The numerical computations have also shown that the conditions given by a set of equations (2) are valid only for some specific cases. In the general case, the matrix in Eq. (1) may be neither Hermitian nor unitary. Therefore, the method for obtaining the ORP from the components of the matrix in Eq. (1) is not obvious as was presumably assumed by Muševič and Śkarabot [14]; this is being discussed in detail here.

The transmitted light, in general, as already stated, is elliptically polarized and depends on the orientation of the molecules (or in the case of a tilted smectic subphase, on the c directors) at the surface of the sample (the first surface from the light source) with respect to the polarization direction of the incident light. This implies that the two arrangements of the c directors given in Figs. 1(a) and 1(b) between the polarizer and the analyzer should give different transmitted intensities with different results of the ORP, despite the fact that the only difference between these two cases is the initial azimuthal angle $\varphi_{0}$ of the $\mathbf{c}$ directors.

In a real chiral smectic homeotropic sample, there is no fixed tilt direction on the substrate, i.e., $\varphi_{0}$ is randomly distributed over the sample area. This is the reason for the observation of the Schlieren textures in thin films [12].

When the condition of pure optical rotation $\lambda \gg \Delta n P$ is satisfied, the effect of the initial azimuthal angle $\varphi_{0}$ on the polarization of transmitted light is negligible. In this case, the sample appears uniform under a polarizing microscope and the optical rotation can be obtained from the orientation of the long axis of the polarization ellipse of the transmitted light [15]. In that case alone, the output light is nearly linearly polarized.

The expression for the angle between the long axis of an ellipse and the $x$ axis is (Ref. [19], p. 8 or Ref. [20], p. 27) given by

$$
\tan (2 \alpha)=\frac{2\left|T_{x}\right|\left|T_{y}\right|}{\left|T_{x}\right|^{2}-\left|T_{y}\right|^{2}} \cos \left[\arg \left(T_{x}\right)-\arg \left(T_{y}\right)\right],
$$

where $T_{x}$ and $T_{y}$ are the same as in Eq. (1). The arg implies the argument of a complex number. If electric field of the incident wave is parallel to the $x$ axis, i.e., $E_{x}=1, E_{y}=0$, then the angle $\alpha$ represents the optical rotation of the sample.

But in the case of the $\mathrm{SmC}_{\mathrm{F} 11}$ * and the $\mathrm{SmC}_{\mathrm{F} 12}$ * phases the nonuniformity of the texture is clearly visible (Fig. 7), as the condition $\lambda \gg \Delta n P$ is not being met. In this case, a different approach needs to be used for simulating the experimentally obtained signal.

The transmittance measured by the setup described above is not from a single geometrical point of the cell. It is an average value of the transmitted light intensity over a field of view of the microscope. We assume that the field of view is large enough and $\varphi_{0}$ can take any value with the same probability (Fig. 7). Therefore, the averaging can be carried out over the initial azimuthal angle $\varphi_{0}$. In the case of the light intensity over a sample area, measured as a function of the angle of polarization by the photodiode, we can write

$$
I(\gamma)=\frac{1}{2 \pi} \int_{0}^{2 \pi}\left|T_{x}\left(\gamma, \varphi_{0}\right)\right|^{2} d \varphi_{0} .
$$

In Eq. (4), the component $T_{y}\left(\gamma, \varphi_{0}\right)$ is absent as this is suppressed by the analyzer. $T_{x}\left(\gamma, \varphi_{0}\right), T_{y}\left(\gamma, \varphi_{0}\right)$ can be obtained from the elements of the matrices of Eq. (1) using the rotation matrices as given below

$$
\begin{aligned}
\left(\begin{array}{c}
T_{x}\left(\gamma, \varphi_{0}\right) \\
T_{y}\left(\gamma, \varphi_{0}\right)
\end{array}\right)= & \left(\begin{array}{cc}
\cos \left(\varphi_{0}\right) & -\sin \left(\varphi_{0}\right) \\
\sin \left(\varphi_{0}\right) & \cos \left(\varphi_{0}\right)
\end{array}\right)\left(\begin{array}{ll}
t_{x x} & t_{y x} \\
t_{x y} & t_{y y}
\end{array}\right) \\
& \times\left(\begin{array}{cc}
\cos \left(\varphi_{0}\right) & \sin \left(\varphi_{0}\right) \\
-\sin \left(\varphi_{0}\right) & \cos \left(\varphi_{0}\right)
\end{array}\right)\left(\begin{array}{c}
\cos (\gamma) \\
\sin (\gamma)
\end{array}\right) .
\end{aligned}
$$

Here, $\gamma$ is the angle between the $O x$ axis and the polarization direction of the incident light (Fig. 1). In this case, the components of the incident electric field vector in the laboratory coordinate system can be presented as $E_{x}=\cos (\gamma), E_{y}$ $=\sin (\gamma)$. During the experiment, the polarizer rotates at a constant angular velocity, i.e., the parameter $\gamma$ changes linearly with time. The matrix coefficients $t_{x x}, t_{x y}, t_{y x}, t_{y y}$ are obtained using the Berreman's method, which is a $4 \times 4$-matrix method. We may note that Eq. (1) is just an abbreviated form of the relation between the incident and the transmitted wave given by Eq. (87) and (88) in Ref. [18]. Similar notation can also be found in Ref. [20] where another modification of the $4 \times 4$-matrix method is described. This approach is possible since the $4 \times 4$ matrix is reduced to 2 $\times 2$ matrix by using the boundary conditions at the interface between the liquid crystal and the air. In general, the coefficients so found do not satisfy the conditions given by a set of Eq. (2), however, the coordinate rotation matrices can still be applied to avoid the entire procedure of the computations using the $4 \times 4$-matrix method to calculate these coefficients being repeated for every value of $\varphi_{0}$ in order to enable the analytical integration in Eq. (4) to be carried out. Equation (5) is based on calculating the transmitted intensity from the incident light using the equation $T=R^{-1}\left(\varphi_{0}\right) t_{i j} R\left(\varphi_{0}\right) E$, where $T$ and $E$ are the transmitted and incident vectors of the transmitted and incident light in the laboratory coordinate system. The matrix elements $t_{i j}$ are calculated using the Berreman matrix method for $\varphi_{0}=0$ in the local coordinate system fixed to $\varphi_{0} \cdot R\left(\varphi_{0}\right)$ and $R^{-1}\left(\varphi_{0}\right)$ being the rotation and inversion rotation matrices, respectively.

The result of integration in Eq. (4) can be expressed as

$$
I(\gamma)=A \cos ^{2}(\gamma)+B \sin ^{2}(\gamma)+C \cos (\gamma) \sin (\gamma),
$$

where the coefficients $A, B$, and $C$ in terms of matrix elements of Eq. (1) are found to be 


$$
\begin{gathered}
A=\frac{3\left|t_{x x}\right|^{2}+3\left|t_{y y}\right|^{2}+\left|t_{x y}\right|^{2}+\left|t_{y x}\right|^{2}+2 \operatorname{Re}\left(t_{x x} t_{y y}^{*}+t_{x y} t_{y x}^{*}\right)}{8}, \\
B=\frac{\left|t_{x x}\right|^{2}+\left|t_{y y}\right|^{2}+3\left|t_{x y}\right|^{2}+3\left|t_{y x}\right|^{2}-2 \operatorname{Re}\left(t_{x x} t_{y y}^{*}+t_{x y} t_{y x}^{*}\right)}{8} \\
C=\frac{\operatorname{Re}\left(t_{x x} t_{y x}^{*}+t_{y x} t_{y y}^{*}-t_{x x} t_{x y}^{*}-t_{x y} t_{y y}^{*}\right)}{2} .
\end{gathered}
$$

The measured intensity $I(\gamma)$ is a biased sine wave. The biased sine wave can be written as follows:

$I(\gamma)=I_{0}^{\prime}\left[\cos ^{2}(\alpha+\gamma) \cos ^{2}\left(\pi \Delta n^{\prime} d / \lambda\right)+\frac{1}{2} \sin ^{2}\left(\pi \Delta n^{\prime} d / \lambda\right)\right]$,

where

$$
\alpha=\frac{1}{2} \tan ^{-1}\left(\frac{C}{B-A}\right)
$$

is the optical rotation of the sample, $I_{0}^{\prime}=A+B$ is the intensity of the transmitted light prior to entering the analyzer. This differs from the intensity of the incident light by the reflectance; and

$$
\frac{\pi \Delta n^{\prime}}{\lambda}=\cos ^{-1}\left(\sqrt{\frac{(A-B)^{2}+C^{2}}{(A+B)^{2}}}\right)
$$

is the effective value of the retardation of the wave produced by the sample. This term accounts for the observation of the Schlieren textures.

Here, the sample is presented as a combination of a phase shifter (retarder) and a rotator. This representation allows for a simulation of the true rotatory power which is not being influenced by $\varphi_{0}$. The effective phase shift was introduced for describing the retardation caused by a nonintegral number of helical pitches in the cell. In order to verify the method of averaging, a few test cases have been investigated and compared with the results reported earlier. With a change in the azimuthal angle $\varphi_{0}$, the angle of the polarization rotation calculated using Eq. (3), where the result depends on $\varphi_{0}$, shows oscillations around the value obtained by Eq. (9). The optical rotational angle $\alpha$, computed using Eq. (9) does not depend on $\varphi_{0}$ since the equation is obtained by averaging over this angle. The ORP is calculated on dividing $\alpha$ by the cell thickness $d$.

Figure 2 presents the simulated results of the ORP for different values of $\varphi_{0}$ and for the same set of parameters as in Ref. [14], their Fig. 2. The values of the initial azimuthal angle, the cell thickness, and the smectic layer thickness were not specified in Ref. [14], results have been confirmed using reasonable values of the cell thickness of ten helical pitches and the smectic layer thickness of $3 \mathrm{~nm}$. When the initial azimuthal angle is zero and the cell thickness is an integral multiple of the pitch length, the results obtained by using Eq. (9) coincide with those obtained using Eq. (3). But computations for an arbitrary value of $\varphi_{0}$ or cell thickness lead to significant disagreement.

Figure 3 represents the wavelength dependence of the op-

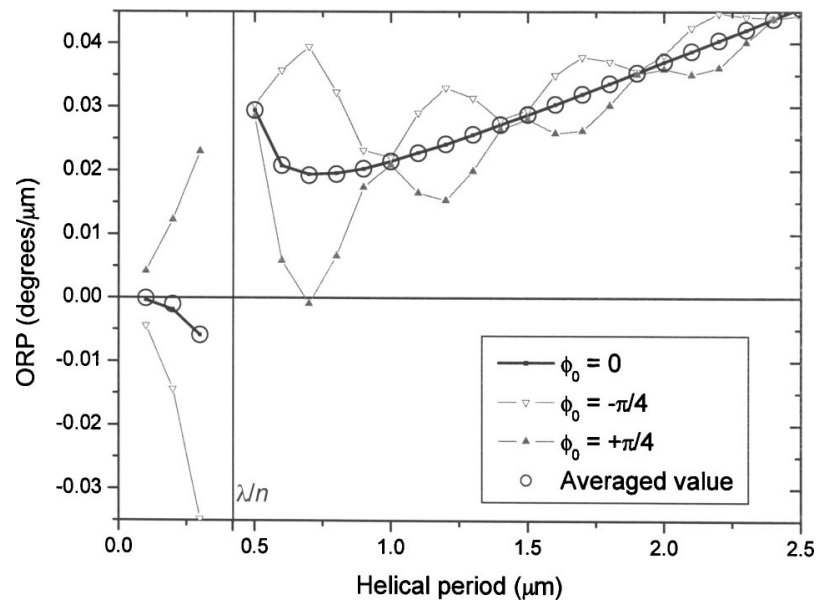

FIG. 2. Comparison of the optical rotatory power of ferroelectric smectic- $C^{*}$ phase, calculated from Berreman's $4 \times 4$-matrix approach for different values of $\varphi_{0}$ and the averaged value as a function of helical period. The tilt angle is $16.2^{\circ}$, whereas the eigenvalues of the dielectric tensor are $\varepsilon_{1}=\varepsilon_{2}=2.28$ and $\varepsilon_{3}$ $=2.89$, the wavelength of light is $632.8 \mathrm{~nm}$.

tical rotation by $\mathrm{Sm} C^{*}$ homeotropic sample of $30 \mu \mathrm{m}$ thickness with helical pitch length of $3 \mu \mathrm{m}$, the eigenvalues of the dielectric tensor of $2.28,2.28,2.89$, and the tilt angle of $26^{\circ}$ are used. The values of the ORP simulated for the other chiral tilted phases with the same value of the helical pitch, tilt angle, and the unit-cell-averaged dielectric tensor, will practically be the same when the smectic layer thickness (i.e., the step of the computing in the matrix method being taken is $3 \mathrm{~nm}$ ) is much shorter than the wavelength of the incident light. The absolute value of the angle between the long axis of an ellipse and the $x$ axis, Eq. (3), is either lower or higher than that of the optical rotation obtained by Eq. (9) depending on the initial azimuthal angle selected for calculations. The results of Eq. (9) approximately agree with those obtained from the de Vries's expression modified for the

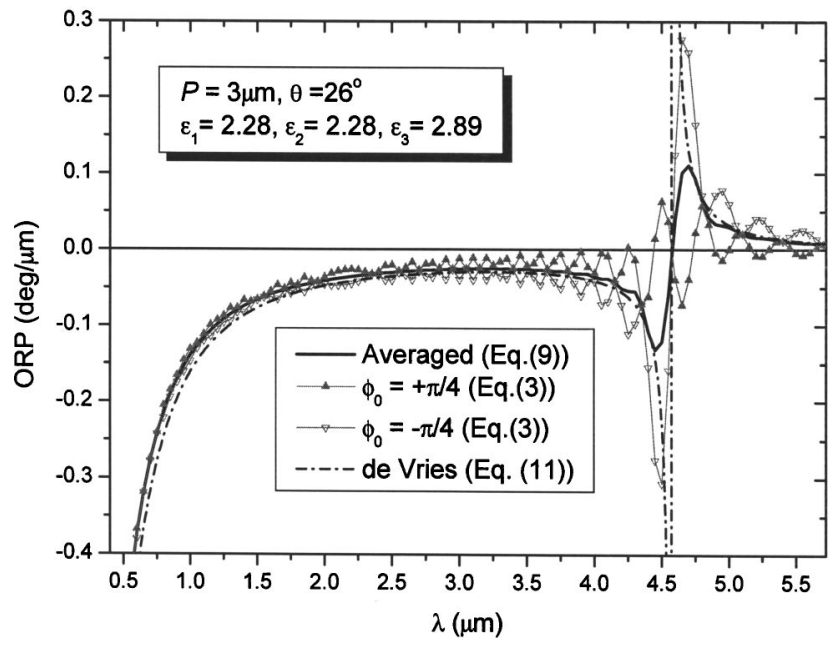

FIG. 3. Wavelength dependence of the ORP in $\mathrm{SmC}$ * phase, calculated from de Vries's analytical solutions of the wave equation and Berreman's $4 \times 4$-matrix approach with the use of Eq. (9) in comparison with the apparent optical rotation obtained from Eq. (3) for different values of $\varphi_{0}$. 


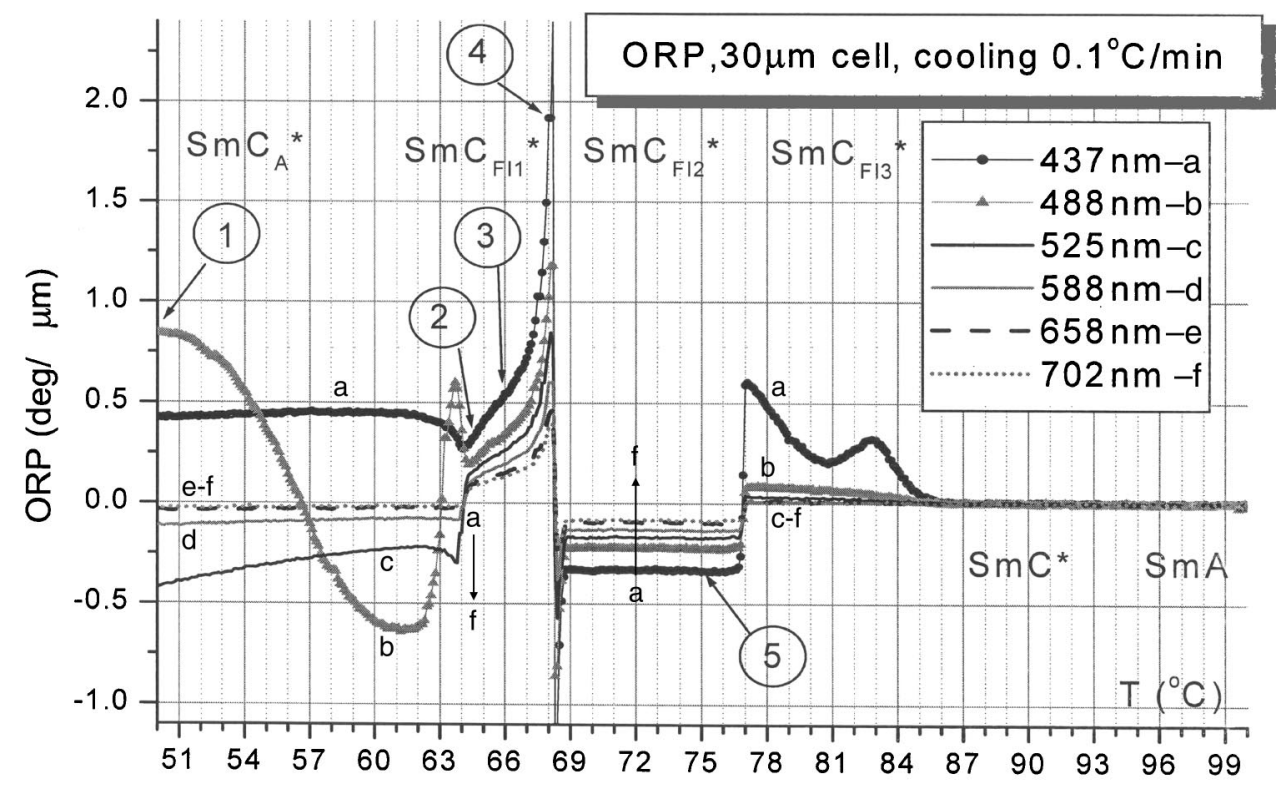

FIG. 4. Optical rotatory power of $(S)-11 \mathrm{OF} 1 \mathrm{M} 7$.

tilted smectic phases (Ref. [21] p. 277, [8]) except close to the selective reflection band:

$$
\begin{gathered}
\Psi=\frac{2 \pi}{8 P}\left(\frac{n_{e}^{2}-n_{o}^{2}}{n_{e}^{2}+n_{o}^{2}}\right)^{2} \frac{1}{\lambda^{\prime 2}\left(1-\lambda^{\prime 2}\right)}, \\
\lambda^{\prime}=\frac{\lambda}{n P}, \quad n=\left(\frac{n_{e}^{2}+n_{o}^{2}}{2}\right)^{1 / 2}, \\
n_{e}^{2}=\frac{\varepsilon_{1} \varepsilon_{3}}{\varepsilon_{1} \cos ^{2} \theta+\varepsilon_{3} \sin ^{2} \theta}, \quad n_{o}=\sqrt{\varepsilon_{2}},
\end{gathered}
$$

where $P$ is the helical pitch, $\lambda$ is the wavelength in vacuo, $n_{e}$ and $n_{o}$ are the refractive indices parallel and perpendicular to the director, $\varepsilon_{1}, \varepsilon_{2}$, and $\varepsilon_{3}$ are the principal values of the dielectric tensor for the chiral tilted smectics at the optical wavelengths, and $\theta$ is a tilt angle of the director with respect to the smectic layer normal. The de Vries's formula is applicable to the case of an infinite media and, therefore, the initial azimuthal angle does not enter in the equation in this case. The value of the ORP diverges at the critical wavelength and at the other wavelengths the absolute value of the ORP obtained from Eq. (11) is slightly overestimated in comparison with those obtained by the exact $4 \times 4$-matrix method for a cell of finite thickness. By decreasing the helical pitch (i.e., approaching the condition of pure optical rotation $\lambda \gg \Delta n P)$, the differences between the results from Eqs. (9) and (3) become small as should be the case.

On varying $\varphi_{0}$, the results obtained from Eq. (3) oscillate around those obtained from Eq. (9). This ensures that our method and the software are correct.

\section{EXPERIMENTAL RESULTS}

The ORP is obtained by dividing the phase part of the experimental biased curved $(2 \alpha)$ by two times the cell thickness (i.e., $2 d$ ) as shown in Eq. (9). Figure 4 gives the experimental results of the optical rotatory power in
$(S)$-11OF1M7. The rotatory power behavior at the temperatures designated by circled numbers from 1 to 5 will be discussed in detail below. The circled numbers correspond to those given in Fig. 8-10.

The value of the ORP in the $\mathrm{SmC} C^{*}$ phase $\left(88^{\circ} \mathrm{C}-85^{\circ} \mathrm{C}\right)$ is found to be extremely low. This can be explained by a very small value of the helical pitch in this phase.

In the $\mathrm{Sm} C_{\mathrm{F} 13}{ }^{*}$ phase $\left(85^{\circ} \mathrm{C}-76.9^{\circ} \mathrm{C}\right)$, the absolute value of the ORP becomes significantly large for a wavelength of $437 \mathrm{~nm}$. With an increase in the wavelength of the light, the optical activity decreases rapidly. The ORP for a wavelength of $437 \mathrm{~nm}$ changes with temperature and for some sets of measurements even a change in the sign of the ORP can be observed. This means that the critical wavelength of the optical rotation in this phase is very close to $437 \mathrm{~nm}$. In this wavelength range, a small change in the helical pitch or the birefringence of a unit cell with temperature can cause a large change in the absolute value to the extent that a change in the sign of the ORP can also occur.

To confirm the dependence of the helical pitch on temperature, the helical pitch was investigated in an independent experiment through measurements of the selective reflection of light. The maximum of the selective reflection has been found for a wavelength of $437.5 \mathrm{~nm}$. This is in good agreement with the results of the ORP measurements. However, the structure of the $\mathrm{SmC}_{\mathrm{F} 13}$ * (FiLC) phase is still among the topics for further investigations.

In the $\mathrm{SmC}_{\mathrm{F} 12}{ }^{*}$ phase $\left(76.9^{\circ} \mathrm{C}-68.3^{\circ} \mathrm{C}\right)$, the ORP practically does not change with temperature. Meanwhile, it increases with a decrease in the wavelength of light. Unlike the $\mathrm{SmC}_{\mathrm{F} 13} *$ and the $\mathrm{SmC}_{A}$ phases, the optical rotation for the long wavelengths of light in the $\mathrm{SmC}_{\mathrm{F} 12}$ * phase $(702 \mathrm{~nm})$ is significantly large. In this case, the helical pitch is larger than the longest wavelength used for the measurements. This was confirmed by the observation of a thick planar cell (see the inset in Fig. 9), where the pitch length was found to be 1.8 $\mu \mathrm{m}$. A sharp peak in the absolute value of the ORP near the 


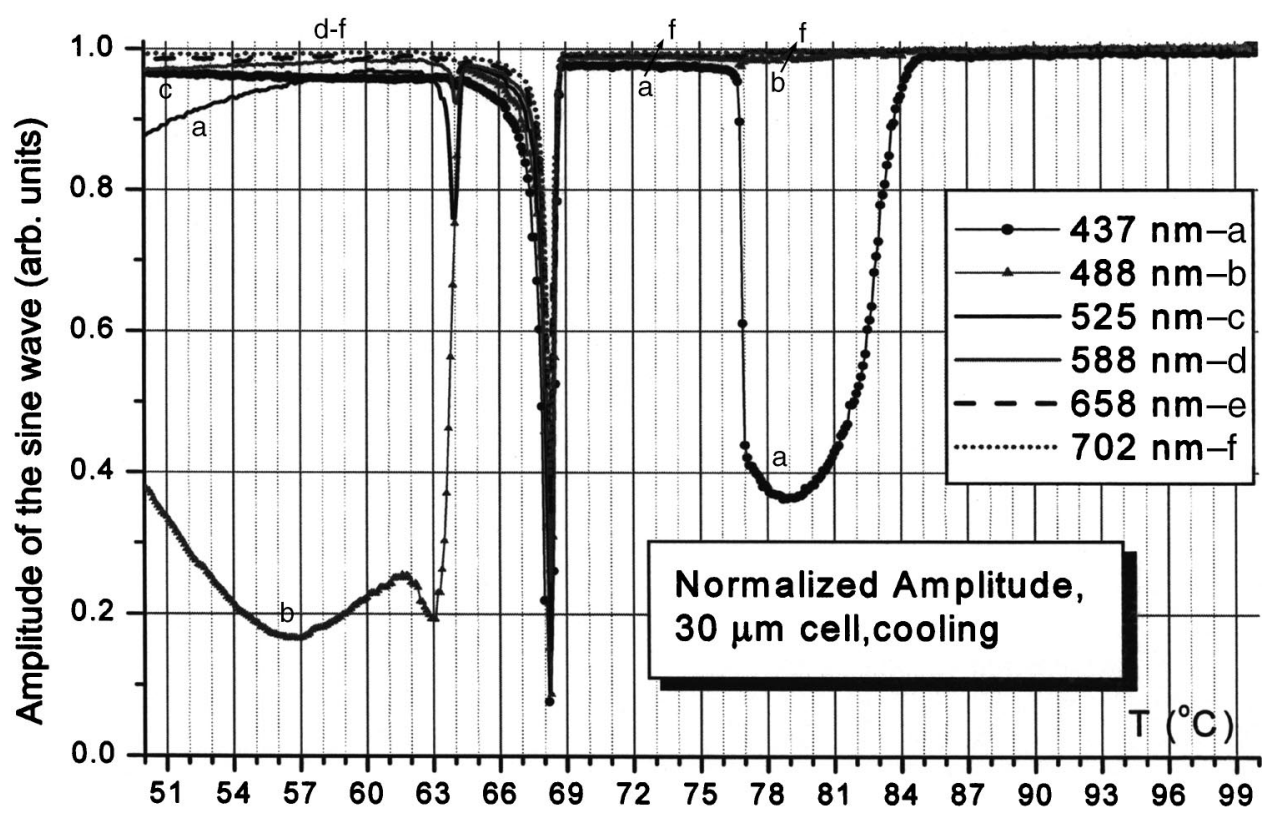

FIG. 5. The amplitude of the light intensity oscillations during the ORP measurements.

phase transition from the $\mathrm{SmC}_{\mathrm{F} 12}$ * to the $\mathrm{SmC} C_{\mathrm{F} 11}$ * phase can be explained either by the pretransitional helical unwinding or by a coexistence of these two phases.

In the $\mathrm{SmC}_{\mathrm{F} 11^{*}}$ phase $\left(64^{\circ} \mathrm{C}-76.9^{\circ} \mathrm{C}\right)$, such as in the $\mathrm{Sm}_{\mathrm{F} 12} *$ phase, the ORP has a significant value and decreases with the wavelength of light. In this case, the helical pitch is also suggested to be longer than $702 \mathrm{~nm}$. But the experiments on the direct measurements of the helical pitch were beset with significant experimental difficulties. The observation of a thick planar cell provides no obvious periodicity in the disclination lines (see the inset in Fig. 10). This can be explained either by a large dependence of the helical pitch in the $\mathrm{SmC}_{\mathrm{F} 11}$ * phase on temperature and/or by a very long (tens of hours) relaxation time of the texture in a planar cell. In this case, even at a moderate cooling rate of $0.1^{\circ} \mathrm{C} /$ min, the measurements of the helical pitch with this method will be impossible, as discussed later.

The key feature of the ORP behavior in the $\mathrm{SmC} C_{\mathrm{F} 11}$ * phase is an increase in its absolute value with temperature. The optical activity increases significantly in the vicinity of the $\mathrm{SmC}_{\mathrm{F} 12} *-\mathrm{SmC}_{\mathrm{F} 11}$ * phase transition. This confirms the observation of the temperature dependence of the helical pitch in the $\mathrm{SmC}_{\mathrm{F} 11}$ * phase.

In the $\mathrm{SmC}_{A}{ }^{*}$ phase (below a temperature of $64{ }^{\circ} \mathrm{C}$ ), the situation is similar to that in the $\mathrm{SmC}_{\mathrm{F} 13}$ * phase. The sign of the ORP at $50{ }^{\circ} \mathrm{C}$ is the same for the long wavelengths $(702$, 658,588 , and $525 \mathrm{~nm}$ ) but opposite for the short wavelengths of (437 and $488 \mathrm{~nm}$ ). According to Fig. 3, this implies that the wavelength of the selective reflection band is located between 448 and $525 \mathrm{~nm}$. The independent investi-

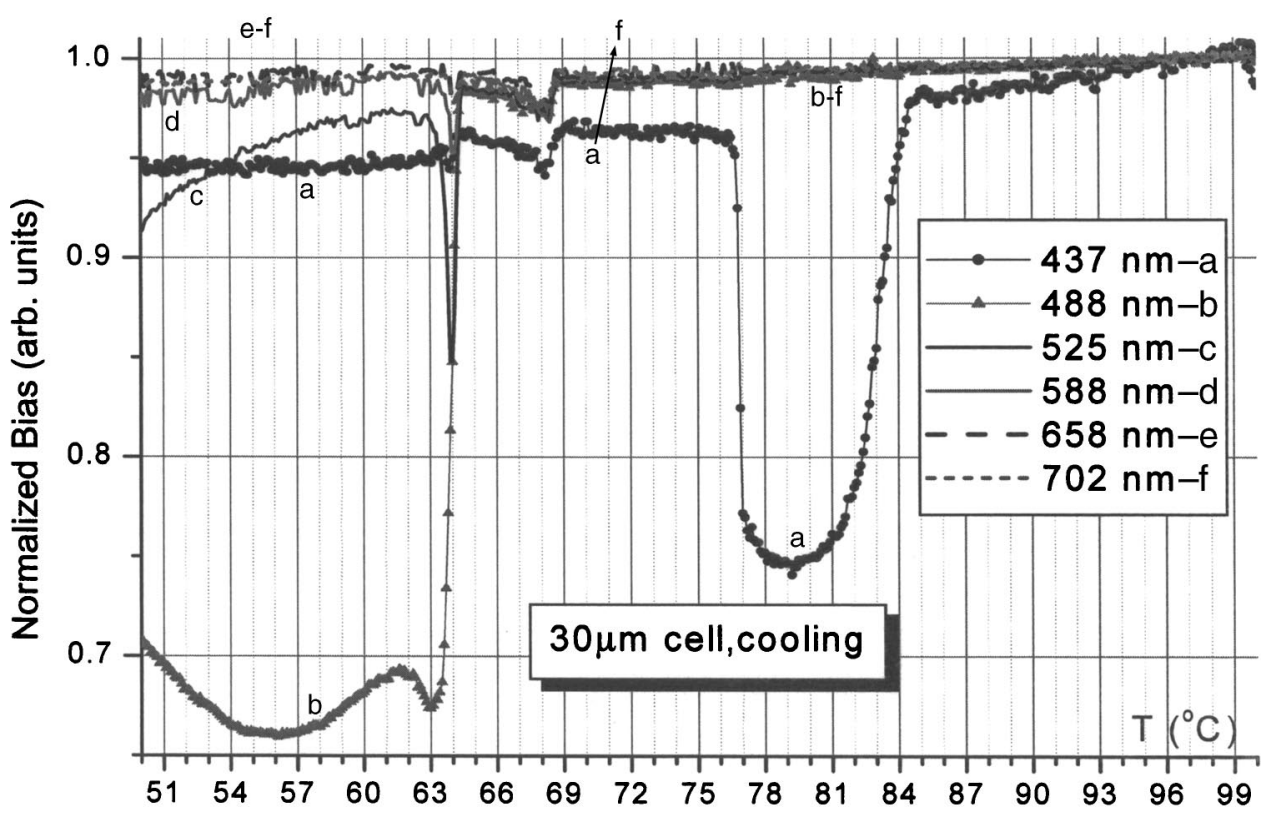

FIG. 6. Offset of the sine wave signal measured during the ORP experiment. 


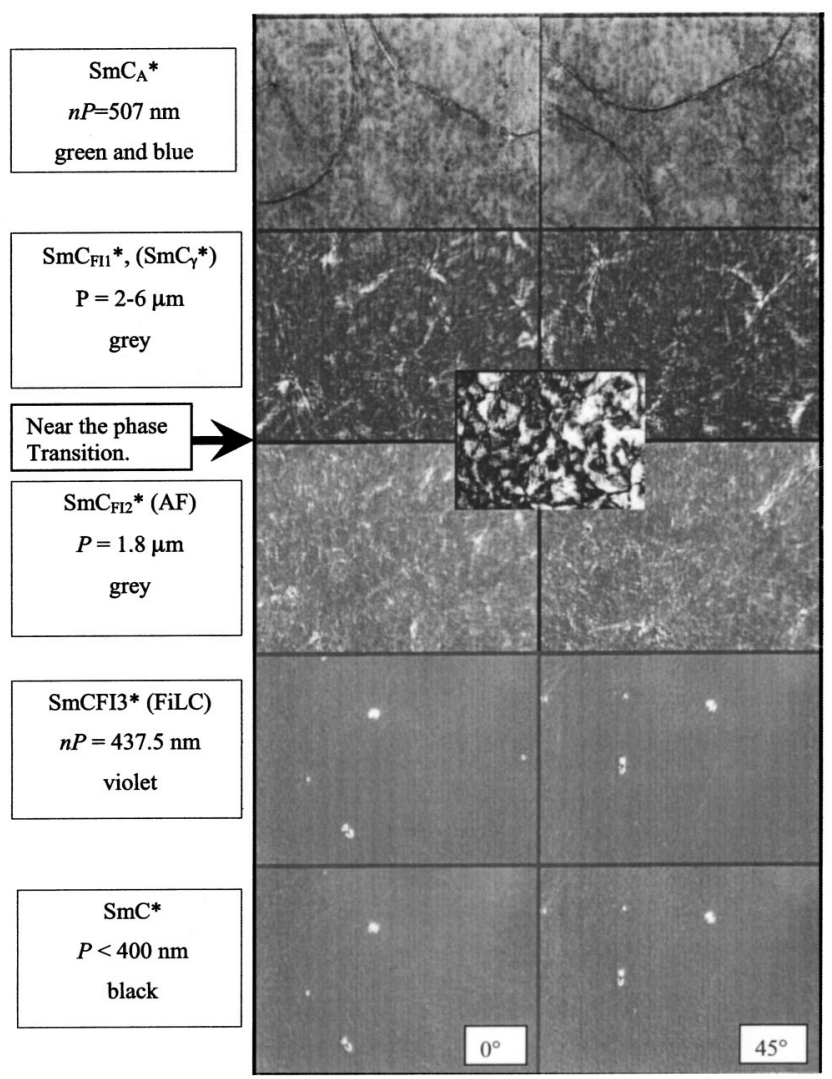

FIG. 7. The textures at crossed polarizers. Smectic phases obtained during slow cooling of a $30-\mu \mathrm{m}$ homeotropic cell, $(S)$-11OF1M7

gations of the selective reflection by UV-visible spectrophotometer shows $n P=507 \mathrm{~nm}$ at a temperature of $50{ }^{\circ} \mathrm{C}$. The helical pitch decreases slightly with temperature and at $T$ $=56.6^{\circ} \mathrm{C}, n P=488 \mathrm{~nm}$. At this temperature, the ORP measured at the wavelength of $488 \mathrm{~nm}$ changes its sign.

The sign of the ORP in the $\mathrm{Sm} C_{\mathrm{F} 12}$ * phase is opposite to that in the $\mathrm{SmC}_{\mathrm{F} 11}$ * phase for all wavelengths. This means that the handedness of the chiral structure is changed during the transition from the $\mathrm{Sm} C_{\mathrm{F} 12}$ * to the $\mathrm{Sm} C_{\mathrm{F} 11}$ * phase. This fact allows for these two phases to be distinguished from each other. It may be emphasized that the pitch inversion inside a given phase usually occurs in liquid crystalline materials that are mixtures of liquid crystal (LC) compounds with opposite handedness. This can mean that the temperature dependences of the pitches for the left-handed and the right-handed compounds are different. In this case, the pitch goes to infinity at the inversion temperature and decreases when the temperature moves away from the inversion temperature. Results on the temperature dependence of the ORP are symmetrical with respect to the inversion temperature; that is, the ORP will have approximately the same absolute values but opposite signs below and above the inversion temperature. Our ORP results do not demonstrate this kind of behavior inside a single phase of the investigated compound. The inversion in the sign of the ORP at the transition from the $\mathrm{SmC}_{\mathrm{F} 12}$ * to the $\mathrm{SmC}_{\mathrm{F} 13}$ * is connected with increase in the pitch length in the $\mathrm{SmC} C_{\mathrm{F} 12}$ * phase and not with the pitch inversion. In our case, we have an optically pure compound.

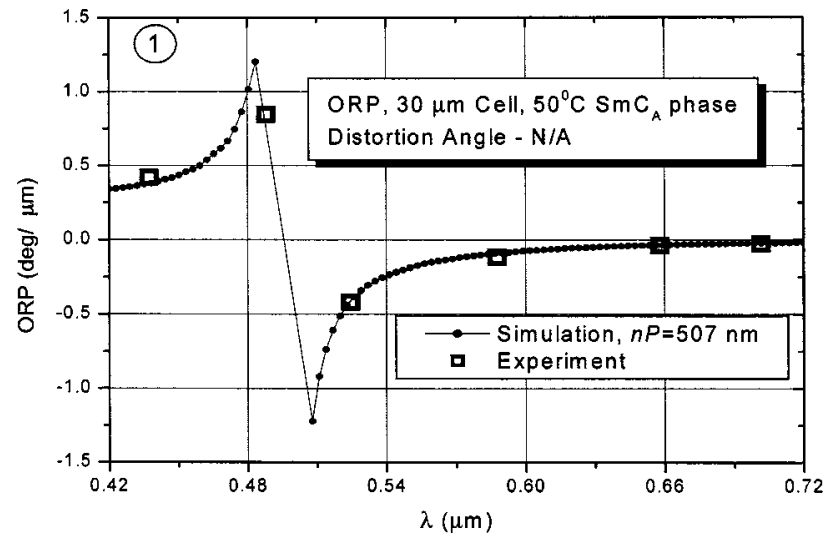

FIG. 8. The optical rotatory power measured in the $\mathrm{SmC}_{A}{ }^{*}$ phase and its simulation by the $4 \times 4$-matrix method.

The existence of the phases $\mathrm{Sm}_{\mathrm{F} 13} *, \mathrm{Sm}_{\mathrm{F} 12} *$, and $\mathrm{Sm} C_{\mathrm{F} 11}$ * confirms its optical purity.

Additional parameters were measured during the same experiment. Figures 5 and 6 show the amplitude and the offset of the signal fed to the oscilloscope. Since the photodiode sensitivity and the maximum transmittance of the filters depend on the wavelength, the data are to be normalized to allow for a comparison of the parameters for different wavelengths to occur. For normalization, temperature of the cell at $100{ }^{\circ} \mathrm{C}$ ( $\mathrm{Sm} A^{*}$ phase) was used. In this phase, the texture is uniform and the optical rotation due to a helical structure is absent because the helix in the $\operatorname{Sm} A *$ phase is not present.

The amplitude of the measured sine wave signal depends on the ellipticity of the transmitted light. It reaches its maximum in the case of linearly polarized light and drops to zero when the light transmitted by the sample becomes circularly polarized or not polarized at all.

In the $\mathrm{SmC}_{\mathrm{F} 13} *$ and $\mathrm{SmC}_{A}$ * phases, the amplitude of the sine wave, as seen in Fig. 5, decreases significantly for the light wavelengths of 437 and $525 \mathrm{~nm}$, respectively. Figure 6 shows a similar decrease for the signal offset. Therefore, we can conclude that in this case the total intensity of the light passing through the sample has decreased. This is in good agreement with the results discussed above since the suppressed wavelengths belong to the selective reflection bands of the corresponding phases.

On the contrary, there is a significant drop in the amplitude of the sine wave in the $\mathrm{SmC}_{\mathrm{F} 11}$ * phase near the transition to the $\mathrm{SmC}_{\mathrm{F} 12}$ * phase, while the bias curve does not show any significant change in this region. This means that the nature of decrease in the amplitude in the $\mathrm{SmC}_{\mathrm{F} 11}$ * phase is different. The light is either elliptically polarized or partially polarized due to the random phase shifts introduced by a nonuniform texture. In this case, the term $\cos ^{2}\left(\pi \Delta n^{\prime} d / \lambda\right)$ in Eq. (8) decreases but $I_{0}^{\prime}$ remains the same.

In the $\mathrm{SmC}_{\mathrm{F} 11}$ * phase, the helical pitch increases rapidly as the sample approaches the $\mathrm{SmC}_{\mathrm{F} 12}$ * phase. It seems that in this phase the pitch is too large and the condition of the pure optical rotation $\lambda \gg \Delta n P$ is not fulfilled, i.e., the transmitted light is elliptically polarized. This has been confirmed through microscopic texture observations. Figure 7 shows the textures at crossed polarizers obtained for smectic phases 


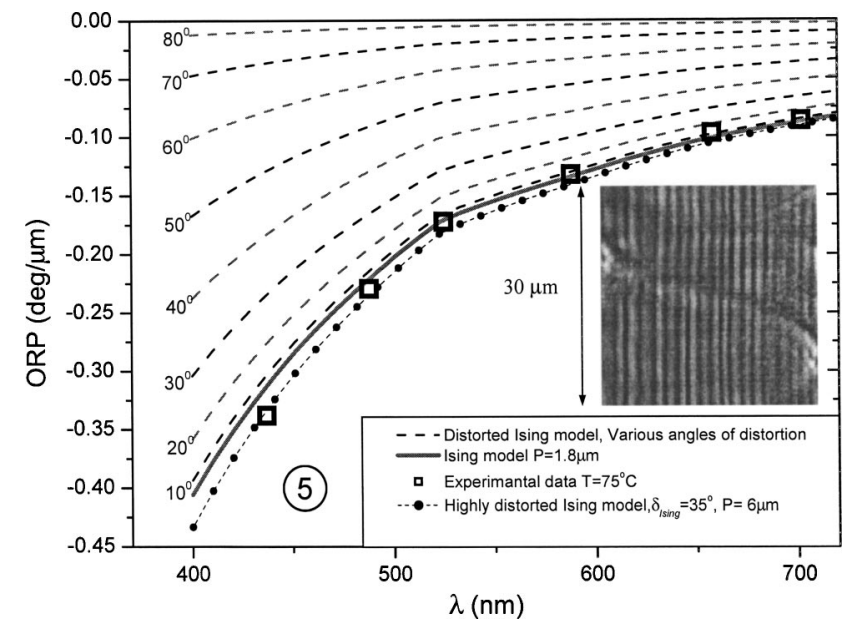

FIG. 9. The ORP measured in the $\mathrm{SmC}_{\mathrm{F} 12}{ }^{*}$ phase and its simulation by the $4 \times 4$-matrix method. Various distortion angles $\delta_{\text {Ising }}$ are presented. The inset shows the view of a thick planar cell as seen through a polarizing microscope.

on cooling the $30-\mu \mathrm{m}$ homeotropic cell of the $(S)$-11OF1 M7 compound. One clearly sees the nonuniformity of the texture for the phases with a long helical pitch. This is the main reason why the method of the ORP measurements involving averaging over the field of view in the microscope had to be applied. When the critical wavelength $n P$ belongs to the visible range, the texture seen through the microscope has the corresponding color (blue/violet for $\mathrm{SmC}_{\mathrm{F} 13} *$ and green/blue for $\mathrm{SmC}_{A} *$ ).

In order to perform a quantitative analysis of the optical rotatory power in the phases, we also need to know the apparent tilt angle and the birefringence of the material. The measurements were performed using the technique described in Refs. [22,23]. In the investigated temperature range, the apparent tilt angle slowly varies with temperature from $\theta$ $=27^{\circ}$ at $T=50^{\circ} \mathrm{C}$ in the $\mathrm{SmC}_{A}$ to $\theta=23.5^{\circ}$ at $T=78^{\circ} \mathrm{C}$ in the $\mathrm{Sm} C_{\mathrm{F} 12}$ * phase.

The change in the birefringence in this temperature range was found to be negligible (about 0.004). Meanwhile, a significant dispersion (from 0.182 at $437 \mathrm{~nm}$ to 0.148 at 702 $\mathrm{nm}$ ) with the light wavelength was found. Therefore, the dispersion of the birefringence has to be considered in the simulations.

\section{DISCUSSION}

For the antiferroelectric $\mathrm{SmC}_{A}$ * phase, the two models under discussion (Ising and clock) provide the same kind of layer to layer progression in the azimuthal angle. The c directors in the adjacent layers are rotated by an angle of $\approx 180^{\circ}$ to each other. Therefore, the data obtained in the $\mathrm{SmC}_{A}$ * phase can be used for confirming results of the measurements and for testing the simulation procedures.

An ORP spectrum at $50{ }^{\circ} \mathrm{C}$ in the $\mathrm{SmC}_{A}{ }^{*}$ phase was simulated numerically using the Berreman's matrix method [18]. The simulated curve and the experimental points are presented in Fig. 8. The parameters used for this simulation were the sample thickness $d=30 \mu \mathrm{m}$; the layer spacing $h$ $=3 \mathrm{~nm}$; the tilt angle of the director $\theta=27^{\circ}$; the indices of

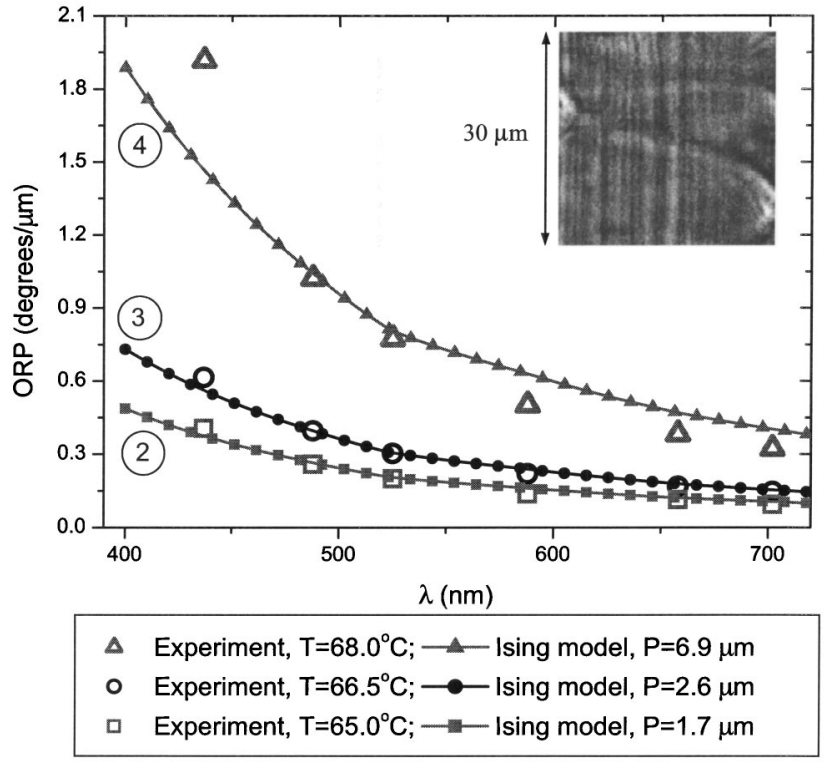

FIG. 10. The ORP measured in the $\mathrm{Sm} C_{\mathrm{F} 11}$ * phase and its simulation by the $4 \times 4$-matrix method. The helical pitch changes with temperature. The inset shows the view of a thick planar cell under polarizing microscope.

refraction $n_{1}=n_{2}=\sqrt{2.0}$, and $n_{3}=n_{1}+\Delta n$ (along the director), where $\theta$ and $\Delta n$ were measured separately. The pitch value of $0.35 \mu \mathrm{m}$ and the refractive indices was found to give the best fit of the experimental data. This is in good agreement with the value of $n P=507 \mathrm{~nm}$ measured by the spectrophotometer (here, $n$ is the average refractive index of the media [15]). We can, therefore, conclude that the method can be used for further investigations.

For both the Ising $[24,8]$ and clock $[25,26]$ models, the structures of the $\mathrm{SmC}_{\mathrm{F} 12}$ * and $\mathrm{Sm} C_{\mathrm{F} 11}$ * phases involve unit cells consisting of four and three smectic layers, respectively. In the Ising model, the azimuthal distribution of the director in the smectic layers forming the unit cell leads to the optical anisotropy of the unit cell in a plane parallel to the smectic layers. This optical anisotropy in the case of a simple clock model [25] is absent due to symmetry in the azimuthal distribution of the director in the unit cell. The simple clock model for the $\mathrm{SmC}_{\mathrm{F} 12}$ * and $\mathrm{Sm} C_{\mathrm{F} 11}$ * phases implies very short-pitch values $P_{\text {short }}$ with periods of four and three layers; therefore, in this case a very small ORP of the order of $P_{\text {short }}^{3} / \lambda^{4}$ should be produced for $P_{\text {short }} \sim 12-16 \mathrm{~nm}$ and $\lambda$ $\sim 500 \mathrm{~nm}$. When in the simple clock model the long-pitch chirality is superimposed, then the structure becomes slightly incommensurate because an additional long-pitch helix slightly increases the increment of the azimuthal angle between the adjacent smectic layers in a unit cell. But even in this case, a very small ORP is expected because of the negligible optical anisotropy of the unit cell in the plane parallel to the smectic layers. This has been confirmed by using the $4 \times 4$-matrix method.

\section{A. Modeling the $\mathrm{SmC} C_{\mathrm{F12}}$ * phase}

The calculations of the ORP in the $\mathrm{SmC}_{\mathrm{F} 12}$ * phase for the structures proposed by the Ising [8] model and for various 
distortion angles from the Ising structure are presented in Fig. 9. The parameters used for these simulations were the same as for the $\mathrm{SmC}_{A}{ }^{*}$ phase, except for the tilt angle of the director and the pitch of the helical structure; these were $\theta$ $=24.4^{\circ}$ and $P=1.8 \mu \mathrm{m}$, respectively.

The distorted clock model with the following symmetric sequence of azimuthal angles given by the equation

$$
\varphi_{j}=\frac{\pi j}{2}-\frac{\delta_{\text {clock }}}{2}+\frac{(-1)^{j} \delta_{\text {clock }}}{2},
$$

where $0 \leqslant j \leqslant 3$ has the following sequence of azimuthal angles: $0,\left(\pi / 2-\delta_{\text {clock }}\right), \pi,\left(3 \pi / 2-\delta_{\text {clock }}\right)$. Where $\delta_{\text {clock }}$ is the distortion angle in the clock model. In terms of the Ising model, the distortion angle will be $\delta_{\text {Ising }}=\pi / 2-\delta_{\text {clock }}$ and the sequence of azimuthal angles takes the following form:

$$
\varphi_{j}=\frac{\pi j}{2}+\frac{\delta_{\text {Ising }}}{2}-\frac{(-1)^{j} \delta_{\text {Ising }}}{2}+\frac{\left[(-1)^{j}-1\right]}{2} \frac{\pi}{2} .
$$

The simple Ising and clock models are the particular cases of the distorted Ising (as well as the distorted clock) model with

$$
\delta_{\text {Ising }}=0 \quad\left(\delta_{\text {clock }}=\pi / 2\right) \text { and } \delta_{\text {Ising }}=\pi / 2 \quad\left(\delta_{\text {clock }}=0\right) \text {, }
$$

respectively. If a long-pitch chirality is added, then the azimuthal angle progression becomes (in terms of the distorted Ising model)

$$
0, \quad \delta_{\text {Ising }}+\varphi_{\mathrm{FI}}, \quad \pi+2 \varphi_{\mathrm{FI}}, \quad \pi+\delta_{\text {Ising }}+3 \varphi_{\mathrm{FI}} .
$$

Here, $\varphi_{\mathrm{FI}}=2 \pi h / P$ is the rotation angle between the adjacent layers and $h$ is the thickness of the smectic layer. This model was named the "highly biaxial model." It describes the ellipsometric data obtained in the $\mathrm{SmC}_{\mathrm{F} 12}$ * and $\mathrm{SmC}_{\mathrm{F} 11}$ * phases of freestanding films [10]. It was shown that the structure is able to explain the resonant $\mathrm{x}$-ray-diffraction results. The simulation of the ORP in this case is presented in Fig. 9 (dashed lines) for the various distortion angles denoted by $\delta_{\text {Ising }}$. When the angle $\delta_{\text {Ising }}$ exceeds $30^{\circ}$, the ORP value decreases rapidly and in the case of the simple clock model ( $\delta_{\text {Ising }}=90^{\circ}$ or $\delta_{\text {clock }}=0^{\circ}$ ), the ORP is $\sim 10^{-6}$ of the ORP value for the Ising model.

Meanwhile, the values of the ORP for the $\delta_{\text {Ising }}=10^{\circ}$ are very close to those when the $\delta_{\text {Ising }}=0^{\circ}$ (thick solid line) and since the difference obtained is within the experimental error, it is practically impossible to determine the actual value of the distortion angle within a range of $10^{\circ}$. Thus, we can conclude that the distortion in the Ising model does not exceed $10^{\circ}$. However, due to the weak dependence of the ORP on the distortion angle when the $\delta_{\text {Ising }}$ is less than $10^{\circ}$, it would appear that the ORP-based techniques will hardly be able to resolve the distortion of the Ising model below this limit.

The larger values of the distortion angle can also provide a good fitting of the experimental data in this wavelength range (the dashed line with black squares represents $\delta_{\text {clock }}$ $=35^{\circ}$ ), but in this case the helical pitch has to be much longer $(6 \mu \mathrm{m})$ than the experimentally measured value. Since this value of the pitch length contradicts the results of the independent measurements, the large distortion angles should be ruled out.

\section{B. Modeling the $\mathrm{SmC} C_{\mathrm{F} 11}$ * phase}

In the case of the $\mathrm{SmC}_{\mathrm{F} 11}$ * phase, the unit cell contains three layers and the following sequence of azimuthal layers should be considered:

$$
0, \pi-\delta_{\text {Ising }}+\varphi_{\mathrm{FI}}, \quad \pi+\delta_{\text {Ising }}+2 \varphi_{\mathrm{FI}}
$$

or in terms of the distorted clock model [14]

$$
0, \quad 2 \pi / 3+\delta_{\text {clock }}+\varphi_{\mathrm{FI}}, \quad 4 \pi / 3-\delta_{\text {clock }}+2 \varphi_{\mathrm{FI}} .
$$

Here, $\delta_{\text {Ising }}=\pi / 3-\delta_{\text {clock }}$.

Figure 10 represents the fitting of the ORP data in the $\mathrm{SmC} C_{\mathrm{F} 11}$ * phase temperature range. The numbers in circles (as well as for Figs. 8 and 9) provides the reference to Fig. 4. The experiment on the independent measurements of the helical pitch in this phase was unsuccessful (see the inset in Fig. 10) and the ORP fitting in the given wavelength range also does not provide an unambiguous value for the pitch length. Therefore, we have to assume the distortion angle in order to determine the pitch from the measured ORP. Since the simple Ising model provides the best fit for the $\mathrm{SmC}_{\mathrm{F} 12}$ * phase, it seems reasonable to try this model for the $\operatorname{Sm} C_{\mathrm{F} 11}$ * phase as well.

The parameters used for these simulations were the same as for the other phases, but the tilt angle was $25.9^{\circ}$ and the helical pitch length was determined by fitting the ORP data.

At the lower temperatures $\left(65^{\circ} \mathrm{C}\right.$ and $\left.66.5^{\circ} \mathrm{C}\right)$, the experimental ORP data (squares and circles in Fig. 10, respectively) provide good agreement with the theoretical model and allows determining the helical pitch. It was found to be $1.7 \mu \mathrm{m}$ at $65^{\circ} \mathrm{C}$ and $2.6 \mu \mathrm{m}$ at $66.5^{\circ} \mathrm{C}$. For higher temperatures, near the $\mathrm{SmC}_{\mathrm{F} 12}$ * to $\mathrm{SmC} C_{\mathrm{F} 11}$ * phase transition temperature, values of the measured ORP deviate significantly from those on the theoretical curve. Varying the distortion angle cannot eliminate this disagreement. A possible explanation may consist of either a coexistence of these two LC phases near the phase transition temperature or at higher temperatures, a long relaxation time of the helix near the phase transition temperature. For the latter, the helix continues to change during the measurements, which leads to different systematic errors for each wavelength. Fukuda et al. $[27,28]$ predicted that the helical pitch is distributed over the sample area due a large free energy of this phase that arises from a nonzero spontaneous polarization $P_{S}$ over a unit cell (zero in the case of the $\mathrm{SmC}_{\mathrm{F} 12}{ }^{*}$ ) and, consequently, the dynamic fluctuations may exist over the pitch length. Our results in the inset of Fig. 10 confirm their prediction.

Now it seems that highly biaxial models $[8,10,26]$ can correctly explain the experimental optical results (both the ORP and the ellipsometric [10] data) and the results of the resonant x-ray diffraction $[6,7]$ in the $\mathrm{SmC} C_{\mathrm{F} 12}$ * and $\mathrm{SmC}_{\mathrm{F} 11}$ * phases. To determine which of these models is actually valid, it is necessary to make more accurate experimental investigations of both the structural and optical properties of the $\mathrm{SmC}_{\mathrm{F} 12} *$ and $\mathrm{SmC}_{\mathrm{F} 11} *$ phases in different AFLC compounds. 


\section{CONCLUSIONS}

The homeotropic alignment proved to be useful in determining the unit cell biaxiality of helical phases. The Schlieren textures cannot be observed in a thick sandwich cell due to its significantly large thickness, the optical rotation caused by a number of helical pitches and nonuniform surface conditions of the sample. However, in the case of a long pitch and for large biaxiality of the unit cell and especially when the condition of pure optical rotation is not met $(\lambda \gg \Delta n P)$, the sample between the crossed polarizers is not uniformly dark. This has clearly been confirmed by our observations. In this case, a special technique has to be designed for measuring and simulating the optical rotation. The measurements of the average intensity of light over the microscopic field of view allow us to obtain the real value of the ORP. The simulated value of the optical rotation should be computed using Eq. (9) and by computing transmitted components using the $4 \times 4$-matrix method.

The results from the described technique of the ORP measurements and simulations are shown to be independent of the random distribution of the initial azimuthal angle over the area of the homeotropic cell.

The simulation carried out using Berreman's $4 \times 4$-matrix method shows good agreement with the experiments for
$\mathrm{Sm}_{A}{ }^{*}, \mathrm{Sm}_{\mathrm{F} 12}$, and $\mathrm{Sm} C_{\mathrm{F} 11}$ * phases. For the simulation, the dependence of the birefringence on the wavelength of light has to be taken into account.

On comparing the independently measured helical pitch length with the one obtained by simulation, we can conclude that in the $\mathrm{SmC}_{\mathrm{F} 12}$ * phase of this compound the distortion angle is lower than the possible experimental error of $\sim 10^{\circ}$.

To obtain the distortion angle in the $\mathrm{SmC}_{\mathrm{F} 11} *$ phase, it is necessary to develop a reliable method for measurements of the helical pitch in this phase. It may be remarked that the predictions of Fukuda et al. [27,28] about the distribution of the helical pitch in this phase over the sample area due to the dynamic fluctuations are being confirmed experimentally. They surmise that the three-layer structure is inherently not stable due to a large free energy that arises from a finite value of the spontaneous polarization over a unit cell $\left(P_{S}\right.$ is about $1 / 3$ of the total $P_{S}$ for the field induced $\mathrm{Sm} C^{*}$ phase at this temperature).

\section{ACKNOWLEDGMENTS}

We thank Professor A. Fukuda and Dr. M. Gorkunov for fruitful discussions. N.M.S. was partly supported by RFBR Grant No. 03-02-17288. Work was funded by the European SAMPA and SFI project (02/IN.1/1031).
[1] S. Pikin, M. Gorkunov, D. Kilian, and W. Haase, Liq. Cryst. 26, 1107 (1999).

[2] M. Yamashita and S. Tanaka, Jpn. J. Appl. Phys., Part 2 37, L528 (1998).

[3] B. Žekš and M. Čepič, Mol. Cryst. Liq. Cryst. Sci. Technol., Sect. A 263, 61 (1995).

[4] P. Bak and R. Bruinsma, Phys. Rev. Lett. 49, 249 (1982).

[5] Yu. P. Panarin and J. K. Vij, in Advances in Liquid Crystals, edited by J. K. Vij, special issue of Adv. Chem. Phys. 113, 271 (2000).

[6] P. Mach, R. Pindak, A.-M. Levelut, P. Barois, H. T. Nguyen, C. C. Huang, and L. Furenlid, Phys. Rev. Lett. 8, 3 (1998).

[7] P. Mach, R. Pindak, A.-M. Levelut, P. Barois, H. T. Nguyen, H. Baltes, M. Hird, K. Toyne, A. J. Seed, J. W. Goodby, C. C. Huang, and L. Furenlid, Phys. Rev. E 60, 6793 (1998).

[8] T. Akizuki, K. Miyachi, Y. Takanishi, K. Ishikawa, H. Takezoe, and A. Fukuda, Jpn. J. Appl. Phys., Part 1 38, 4832 (1999).

[9] A. Fukuda, Y. Takanishi, T. Isozaki, K. Ishikawa, and H. Takezoe, J. Mater. Chem. 4, 997 (1994).

[10] P. M. Johnson, D. A. Olson, S. Pankratz, T. Nguyen, J. W. Goodby, M. Hird, and C. C. Huang, Phys. Rev. Lett. 84, 4870 (2000).

[11] R. Pindak, in Books of Abstracts of the Sixth European Conference on Liquid Crystals, Halle, Germany, edited by $\mathrm{H}$. Kresse (2001) pp. I-9; A. Cady, J. A. Pitney, R. Pindak, L. S. Matkin, S. J. Watson, H. F. Glesson, P. Cluzeau, P. Barois, A.-M. Levlut, W. Caliebe, J. W. Goodby, M. Hird, and C. C. Huang, Phys. Rev. E 64, 050702(R) (2001).

[12] V. P. Panov, S. S. Seomun, N. M. Shtykov, J. K. Vij, and H. T. Nguyen, Ferroelectrics 278, 47 (2002).
[13] N. M. Shtykov, J. K. Vij, and H. T. Nguyen, Phys. Rev. E 63, 051708 (2001).

[14] I. Muševič and M. Škarabot, Phys. Rev. E 64, 051706 (2001).

[15] N. M. Shtykov, J. K. Vij, R. A. Lewis, M. Hird, and J. W. Goodby, Liq. Cryst. 28, 1699 (2001).

[16] Yu. P. Panarin, O. E. Kalinovskaya, J. K. Vij, D. D. Parghi, M. Hird, and J. W. Goodby, Ferroelectrics 244, 183 (2000).

[17] M. Osipov and A. Fukuda, Phys. Rev. E 62, 3724 (2000).

[18] D. Berreman, J. Opt. Soc. Am. 62, 502 (1972).

[19] S. Huard, Polarization of Light (Wiley, New York, 1997).

[20] P. Yeh and C. Gu, Optics of Liquid Crystal Displays (Wiley, New York, 1999).

[21] P. G. de Gennes and J. Prost, The Physics of Liquid Crystals (Clarendon, Oxford, 1993).

[22] B. Park, S. S. Seomun, M. Nakata, M. Takahashi, Y. Takanishi, K. Ishikawa, and H. Takezoe, Jpn. J. Appl. Phys., Part 1 38, 1474 (1999).

[23] V. P. Panov, S. S. Seomun, J. K. Vij, A. Fukuda, J. L. Gayo, and J. M. Otón, in Book of Abstracts of the Eighth International Conference on Ferroelectric Liquid Crystals, FLC-2001, Washington, DC, USA, edited by R. Shashidhar (2001), pp. P1-51.

[24] T. Isozaki, K. Hiraoka, Y. Takanishi, H. Takezoe, A. Fukuda, Y. Suzuki, and I. Kawamura, Liq. Cryst. 12, 59 (1992).

[25] B. Žekš and M. Čepič, Liq. Cryst. 14, 445 (1993).

[26] A.-M. Levelut and B. Pansu, Phys. Rev. E 60, 6803 (1999).

[27] K. Miyachi, M. Kabe, K. Ishikawa, H. Takezoe, and A. Fukuda, Ferroelectrics 147, 147 (1993).

[28] T. Matsumoto, A. Fukuda, M. Johno, Y. Motoyama, T. Yui, S. S. Seomun, and M. Yamashita, J. Mater. Chem. 9, 2051 (1999). 